ARTICLE

https://doi.org/10.1038/s41467-019-10441-4

\title{
Chromoselective access to Z- or E- allylated amines and heterocycles by a photocatalytic allylation reaction
}

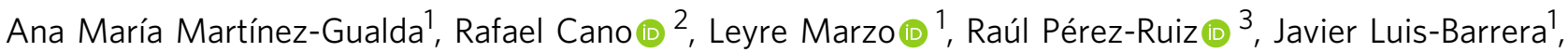
Rubén Mas-Ballesté ${ }^{2,4}$, Alberto Fraile (1) ${ }^{1,4}$, Víctor A. de la Peña O'Shea ${ }^{5}$ \& José Alemán ${ }^{1,4}$

The most useful strategies for the alkylation of allylic systems are related to the Tsuji-Trost reaction or the use of different Lewis acids. Herein we report a photocatalytic approach for the allylation reaction of a variety of nucleophiles, such as heteroarenes, amines and alcohols. This method is compatible with a large variety of pyrroles and indoles, containing different substituents such as electron-withdrawing and electron-donating groups, unprotected nitrogen atoms and bromo derivatives. Moreover, this methodology enables the chromoselective synthesis of Z- or E-allylated compounds. While the use of UV-light irradiation has allowed the synthesis of the previously inaccessible Z-allylated products, $E$-isomers are prepared simply by changing both the light source to the visible region, and the catalytic system. Based on mechanistic and photochemical proofs, laser flash photolysis studies and DFT calculations, a rational mechanism is presented.

\footnotetext{
${ }^{1}$ Organic Chemistry Department, Módulo 1, Universidad Autónoma de Madrid, 28049 Madrid, Spain. ${ }^{2}$ Inorganic Chemistry Department, Módulo 7, Universidad Autónoma de Madrid, 28049 Madrid, Spain. ${ }^{3}$ Departamento de Química, Universitat Politècnica de València, Camino de Vera s/n, 46022 Valencia, Spain. ${ }^{4}$ Institute for Advanced Research in Chemical Sciences (IAdChem), Universidad Autónoma de Madrid, Madrid 28049, Spain.

${ }^{5}$ Photoactivated Processes Unit, IMDEA Energy, Av. Ramón de la Sagra 3C, 28935 Móstoles, Madrid, Spain. Correspondence and requests for materials should be addressed to J.A. (email: jose.aleman@uam.es)
} 
T he preparation of allyl-substituted compounds has attracted a special interest due to their utility as building blocks in organic synthesis $^{1-3}$. The Tsuji-Trost reaction ${ }^{4}$ is one of the most powerful methodologies for the alkylation of allylic systems, which is commonly catalyzed by palladium, and the allylic position is usually activated by a halide, an acetate, or a carbonate (eq. a, Fig. 1) and affords exclusively the $E$-isomer. The high selectivity and the general scope of this reaction makes it one of the most prominent $\mathrm{Csp}^{3}-\mathrm{Csp}^{3}$ bond formation methodologies 5 . Indoles and pyrroles are versatile and useful heterocycles for the synthesis of a large variety of biologically active compounds and natural products ${ }^{6}$. Different authors have reported the allylation of indoles at the $\mathrm{C}-3$ position via the Tsuji-Trost reaction in a racemic manner ${ }^{7-12}$. However, although this methodology is very important, to the best of our knowledge, no photocatalytic approaches for the allylation of heterocycles have been reported so far.

Over the past decade, photocatalysis has emerged as a powerful tool for the construction of new bonds that are difficult to obtain using other established procedures ${ }^{13-31}$. A large number of photocatalytic methodologies have been described for the formation of new $\mathrm{Csp}^{2}-\mathrm{Csp}^{2}$ bonds. In particular, the arylation of (hetero)-aromatic rings, usually pyrroles, under different photocatalytic systems has been recently reported ${ }^{32-40}$. However, one of the major problems related to this photocatalytic arylation is the large excess of the heterocycle required in this reaction (24-40 equiv.). Although the photocatalytic heteroatomatic ring arylation has been extensively studied, the photoallylation of heterocycles remains an elusive process.

We hypothesize that the reduction of the allylic derivative by a photocatalyst with the adequate redox potential would result in the appropriate intermediate, which will allow the functionalization of the allylic position. There are two prerequisites to achieve this goal: (i) the development of a photocatalytic system able to activate the $\mathrm{C}-\mathrm{O}$ bond; (ii) since an unsaturation is present, it is necessary to control the isomerization of the double bond $(Z$ or $E)$.

In this work, we present a chromoselective photocatalytic allylation of heteroaromatic rings, using smooth conditions and short reaction times to access the $Z$ - or $E$-double bonds, depending on the reaction conditions (eq. b, Fig. 1). In addition, mechanistic and photochemical proofs, DFT calculations, and laser flash photolysis studies enabled us to postulate a plausible mechanistic pathway.

\section{Results}

Optimization of the model reaction. Based on the previous photocatalytic arylation reactions ${ }^{32-40}$, we started the screening of the reaction using the acetate allylic derivative 1a and pyrrole $\mathbf{2 a}$ (18 equiv.) in the presence of different photocatalysts 3 under light irradiation (Table 1). Transition-metal-based photocatalysts $(\mathbf{3 a}-\mathbf{3 b})$ failed to promote the formation of the allylated heterocycle (entries 1 and 2). Several photoorganocatalysts with different reductive power $(\mathbf{3 c}-\mathbf{3 f})$ were tested, but only the 10 phenyl-10H-phenothiazine (PTH) (3e) gave the $Z$-allylated pyrrole 4a with low conversion under 420-nm LED irradiation (entries 3-6). Encouraged by these results, we used an irradiation source with a wavelength closer to the maximum absorption of PTH (entry 7). Pleasantly, using a 365-nm LED, 4a was obtained with a $65 \%$ conversion. In the absence of a photocatalyst, light, and both, the allylation did not proceed (entries 8-10), confirming the photocatalytic nature of this transformation. Different solvents were then evaluated, and the best result was obtained using $\mathrm{CH}_{3} \mathrm{CN}$ (entries 7 and 11-14). In order to decrease the amount of heterocycle, the reaction was carried out using 10 and 2 equivalents of $2 \mathbf{a}$ (entries 15 and 16) and $4 \mathbf{a}$ was obtained with a good yield of $58 \%$ in only $3 \mathrm{~h}$, using just two equivalents of the heterocycle. The use of inorganic bases $\left(\mathrm{Na}_{2} \mathrm{CO}_{3}, \mathrm{LiOAc}\right)$ afforded the final product, although with moderate yield, due to the lower solubility of such bases in acetonitrile (entries 17 and 18).

Substrate scope. Having established the best conditions (Table 1, entry 16), we performed the scope of the reaction (Table 2). With $\mathrm{N}$-methyl pyrrole, the allylic derivative $\mathbf{4 b}$ was obtained with a better yield than $4 \mathbf{a}$ and with a similar selectivity for the $Z$-isomer. Other substituents were tolerated at the $N$-atom of the pyrrole (4c and 4d) with excellent $Z / E$ selectivity (up to 96:4) and with a slight decrease for the phenyl derivative 4c. Indoles without protecting groups at the nitrogen were also employed, keeping the high selectivity for the $Z$-isomers, and with better yields than with the pyrroles (compare $\mathbf{4 e}$ and $\mathbf{4 f}$ with $\mathbf{4 a}$ and $\mathbf{4 b}$ ). Electrondonating groups (EDGs) were well tolerated at different positions of the indole ring $(\mathbf{4 g}, \mathbf{4 h}$, and $\mathbf{4 i})$ as well as electron-withdrawing groups (EWG) at the aromatic ring (4j). A methyl substituent next to the indolinic nitrogen (4k) or the reactive C-3 center (4l)

a

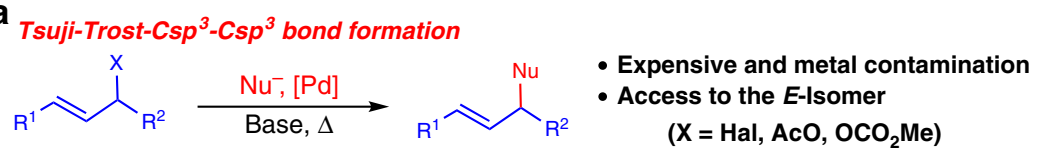

b Photocatalytic allylation

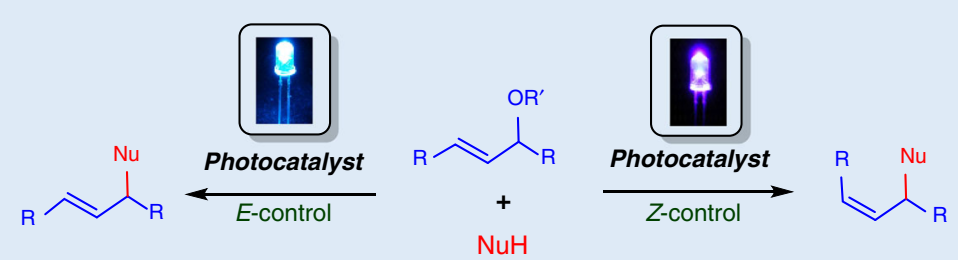

$\mathrm{Nu}=$ Heterocycle, amine or alcohol

- Photocatalytic C-C bond allylic formation

- Smooth conditions \& short reaction times (3 h)
- 2 Equiv. of the nucleophile

- Access to the Z- or E-Isomer

Fig. 1 The photocatalytic allylation reaction. a Precedents in the Tsuji-Trost allylation and $\mathbf{b}$ this work 


\section{Table 1 Optimization of the photocatalytic allylation reaction ${ }^{\mathrm{a}}$}

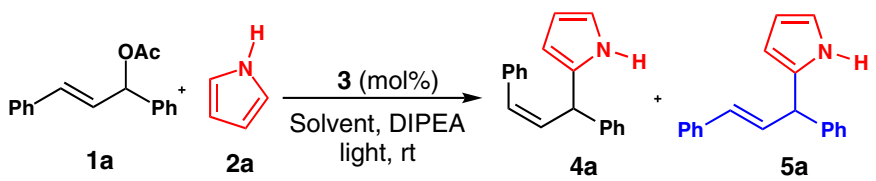

Organic photocatalysts

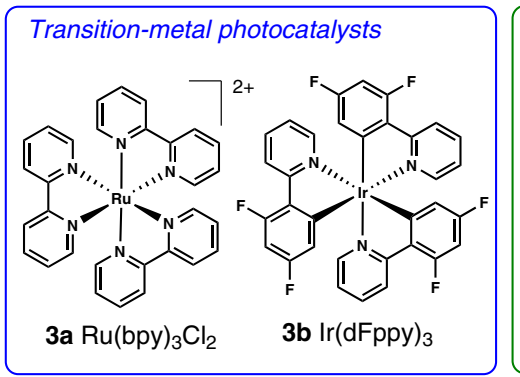

(c)

3c Eosin $Y$<smiles>O=c1c2ccccc2sc2ccccc12</smiles><smiles></smiles>

\section{Entry}

$3(\mathrm{~mol} \%)$

3a (5)

3b (5)

3c (5)

3d (5)

3e (5)

$3 f$ (10)

3e (5)

3e (5)

-

3e (5)

3e (5)

$3 e(5)$

3e (5)

$3 e(5)$

3e (5)

3e (5)

3e (5)

Light (nm)

Solvent

MeCN

MeCN

MeCN

MeCN

$\mathrm{MeCN}$

MeCN

MeCN

MeCN

$\mathrm{MeCN}$

$\mathrm{MeCN}$

DMSO

DMF

Toluene

DCM

MeCN

MeCN

MeCN

$\mathrm{MeCN}$

365

365
365
Pyrrole (equiv.)

18

18

18

18

18

18

18

18

18

18

18

18

18

10

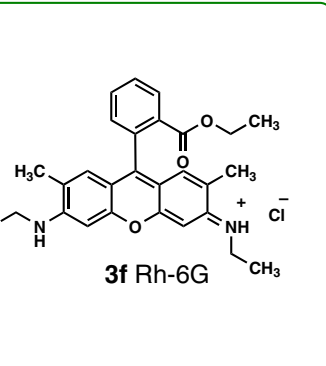

$18^{f}$

${ }^{a}$ Conditions: $\mathbf{1 a}(0.1 \mathrm{mmol}), \mathbf{2 a}$ (see table), DIPEA $(0.5 \mathrm{mmol})$, and catalyst $(\mathrm{mol} \%)$ in the solvent indicated $(1.0 \mathrm{~mL})$

bMeasured by ${ }^{1} \mathrm{H}-\mathrm{NMR}$

'Conversion in the crude mixture

dOptimized conditions highlighted in bold

eReaction carried out under standard conditions but using $\mathrm{Na}_{2} \mathrm{CO}_{3}(0.5 \mathrm{mmol})$ instead of DIPEA

fReaction carried out under standard conditions but using LiOAc $(0.5 \mathrm{mmol})$ instead of DIPEA

did not have a negative influence on the reactivity, obtaining both in very good yields and high selectivity. Remarkably, the presence of $\mathrm{Br}$ at the 5-position $(\mathbf{4 m})$ was also well tolerated under the presence of the high-reducing photocatalyst 3e. The scope of the allylic derivative was also evaluated. Electron-rich (4n, 4o, and $\mathbf{4 p )}$ as well as electron-poor aromatic rings (4q) worked with excellent selectivities (up to $>98: 2$ ). We then studied the influence of the leaving group at the allylic position ( $\mathrm{R}$ group in $1 \mathrm{a})$. The reaction worked with other leaving groups such as benzoate, carbamate, or carbonate, which were suitable for this process. However, the reaction with the hydroxyl group did not proceed, because of its higher reduction potential $(-2.52 \mathrm{~V}$ vs. SCE) compared with the other activated allylic alcohols $(\mathrm{E}=-2.06$ to $-2.35 \mathrm{~V}$ vs. SCE, see Suplementary Note 4 for cyclic voltammetry).

After obtaining these good results with the $Z$-isomer, our next objective was the development of a photocatalytic variant to obtain the corresponding $E$-isomers. To achieve this goal, we analyzed the conditions that avoided the isomerization of the reagent 1a. A sample containing $E-1$ a in $\mathrm{MeCN}$ was irradiated for $3 \mathrm{~h}$ under different reaction conditions (Fig. 2). Without the use of the photocatalyst under 365-nm irradiation, we found a mixture of $60 / 40 E / Z$-1a, while in the presence of $3 \mathbf{e}$, this isomerization to $Z$-1a was complete (Fig. 2). According to theoretical calculations, photosensitization and subsequent isomerization of $E$-1a by the photocatalyst is feasible, while photosensitization and subsequent isomerization of $Z-1$ a cannot take place (see Supplementary Information Fig. 25). The absorption spectra of $E-\mathbf{1 a}$ at the reaction conditions revealed a significant absorption at $365 \mathrm{~nm}$ (see Supplementary Information Fig. 8), while at $420 \mathrm{~nm}$, it was negligible, suggesting that the reaction must be carried out in the visible-light region to avoid isomerization. Under 420-nm irradiation, only $5 \%$ of the $E$-1a was isomerized to the $Z$-isomer after $3 \mathrm{~h}$. Therefore, a photocatalyst with high reduction potential ( $\geq 2.35 \mathrm{~V}$ vs. SCE) and absorption in the visible-light region is required. The phenoxazine $\mathbf{3 g}$, that meets all these criteria $^{41}$, resulted in only a small amount of $Z-1$ a at $420-\mathrm{nm}$ irradiation after $3 \mathrm{~h}$ (Fig. 2). Therefore, under these conditions (using photocatalyst $\mathbf{3 g}$ and $420-\mathrm{nm}$ irradiation), it should be possible to avoid the isomerization step and selectively form $E$ allylated products 5 . 


\section{Table 2 Scope of the allylation reaction for the synthesis of Z-isomers with pyrroles and indoles under catalyst $3 \mathrm{e}^{\mathrm{a}, \mathrm{b}}$}
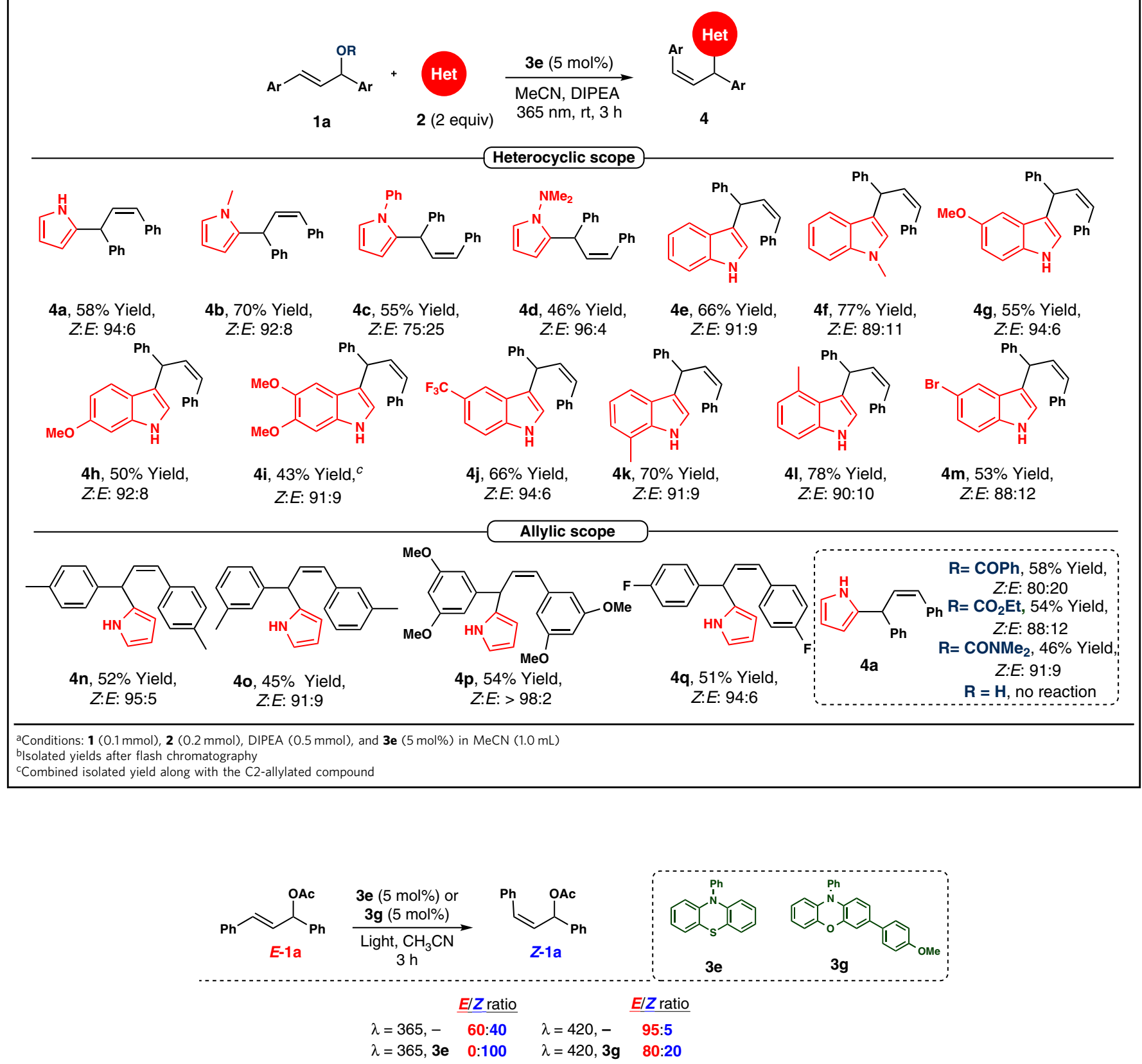

Fig. 2 Isomerization studies. Isomerization proofs of $E-\mathbf{1 a}$ under different catalysts ( $\mathbf{3 e}$ and $\mathbf{3 g}$ ) and different irradiation wavelengths

To our delight, when carrying out the reaction between the allylic derivative $\mathbf{1 a}$ and pyrrole (2a) in the presence of the photocatalyst $\mathbf{3 g}$ under 420 -nm irradiation, the allylated product $E-5$ a was obtained with a good yield as the major isomer (Table 3). Other $\mathrm{N}$-substituted pyrroles were also employed and maintained the same selectivity $(\mathbf{5 b}-\mathbf{5 c})$. Only compound $\mathbf{5 d}$ was obtained as a complex mixture. The reaction with indoles afforded even better yield and selectivity than pyrroles (5e and 5f). Unprotected indolinic nitrogen as well as different substituents were tolerated, from EDGs $(\mathbf{5 g}-\mathbf{i})$ to EWGs $(\mathbf{5 j})$, methyl $(5 \mathbf{k}-\mathbf{1})$, or bromo derivatives $(5 \mathrm{~m})$, obtaining in all cases good yields (67-92\%) and excellent selectivities (up to $>98: 2$ ). The isomerization of the final product $5 \mathbf{e}$ under 420 -nm irradiation was also studied, obtaining a $Z / E$ mixture $30 / 70$ after $3 \mathrm{~h}$ of irradiation, without the photocatalyst, while in the presence of the photocatalyst 3g, a $Z / E$ mixture $20 / 80$ was obtained. The final product is present in the reaction in higher concentrations only after $2 \mathrm{~h}$ of reaction. Therefore, the irradiation time is not enough to produce its isomerization, which explains the obtention of the E-isomer as the major one.

Mechanistic studies. The proposed reaction mechanism is outlined in Fig. 3a. After light absorption by the photocatalyst under LED irradiation $(\lambda=365$ or $420 \mathrm{~nm})$, single-electron transfer (SET) takes place from its $S_{1}$ excited state $\left(E_{\mathrm{S} 1}=3.2 \mathrm{eV}\right.$, see Supplementary Information Figs. 11 and 12) to 1a. Steadystate and time-resolved fluorescence quenching studies in the 
Table 3 Scope of the allylation reaction for the synthesis of E-isomers with pyrroles and indoles under catalyst $3 \mathrm{~g}^{\mathrm{a}, \mathrm{b}}$

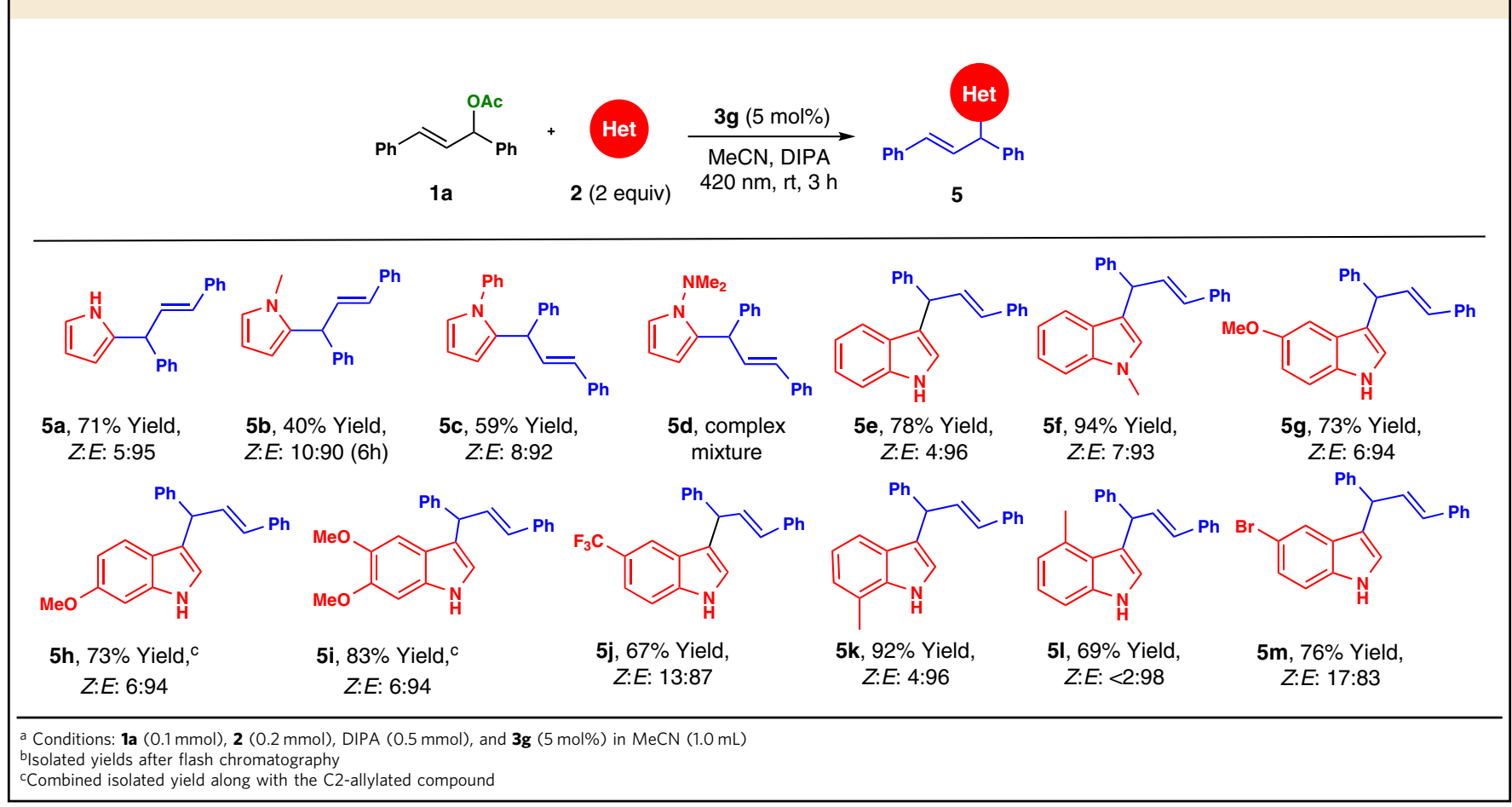

presence of 1a afforded a quenching rate constant of $k_{\mathrm{q}}\left(\mathrm{S}_{1}\right)=$ $4.7 \times 10^{9} \mathrm{M}^{-1} \mathrm{~s}^{-1}$ (see Supplementary Information Fig. 9a), indicating that the radical ion pair $\left(\mathbf{P C}^{\cdot+}+\mathbf{1 a}^{\mathbf{0}^{-}}\right)$formation occurs at nearly diffusion rate. In addition, SET from the excited singlet state would be an exergonic process, taking into account the free energy change $\left(\Delta G_{\mathrm{ET}}=-4.0 \mathrm{kcal} \mathrm{mol}^{-1}\right)$ associated with the electron transfer (see Supplementary Note 4 for Rehm-Weller equation).

Importantly, photooxidation of DIPEA $\left(E_{\mathrm{ox}}=0.94 \mathrm{~V}\right.$ vs. SCE $)^{42}$, DIPA $\left(E_{\text {ox }}=1.17 \mathrm{~V} \text { vs. SCE }\right)^{43}$, or pyrrole $\left(E_{\text {ox }}=1.04\right.$ $\mathrm{V}$ vs. SCE $)^{44}$ by PC $S_{1}$ excited state could not occur, taking into account the oxidation power of $\mathbf{3 e}$ and $\mathbf{3} \mathbf{g}\left(E_{\left(\mathrm{Pc}^{\star} / \mathrm{Pc} \cdot-\right)}\right)=$ $-0.3 \mathrm{~V}$ vs. SCE for $\mathbf{3 e}$ and $\mathbf{3 g}$, see Supplementary Note 4$)$, and was further confirmed by fluorescent-quenching studies (see Fig. 3b). The fate of such reduced species has been investigated by DFT calculations, considering both $Z$ - and $E$-isomers (Fig. 3d). Initial single-electron transfer process from the photocatalyst $(\mathbf{P C})$ to $\mathbf{1 a}$ generates the radical cation $\mathbf{P C}^{+}+$and the radical anion $\mathbf{1} \mathbf{a}^{\cdot-}$, that evolves through the $\mathrm{C}-\mathrm{O}$ bond scission to afford acetate anion and the radical intermediate $\mathbf{I}$ (INT I). This step is a very exergonic process $(-22$ or -21.3 $\mathrm{kcal} \mathrm{mol}^{-1}$ ) and proceeds through a very shallow kinetic barrier $\left(E_{\mathrm{a}}=1.4\right.$ or $\left.3.2 \mathrm{kcal} \mathrm{mol}^{-1}\right)$. Then, the oxidation of INT I by the oxidized photocatalyst $\left(\mathbf{P C}^{+}\right)$, results in the regeneration of the photocatalyst (PC) and formation of a carbocationic intermediate II (INT II) (Fig. 3d). Such electron transfer is calculated as a thermodynamically favorable process $\left(-8.7\right.$ or $\left.-11.8 \mathrm{kcal} \mathrm{mol}^{-1}\right)$. The calculated energetic barriers for $E$ to $Z$ isomerizations for radical or carbocation intermediates I and II rule out this process from such transient species (see Supplementary Information Fig. 28). Formation of this carbocation INT II was experimentally confirmed, carrying out the reaction in the presence of $\mathrm{H}_{2} \mathrm{O}^{18}$ as the nucleophile obtaining the isotopically labeled compound 7 (Fig. 3c). In addition, when two nonsymmetric allylic derivatives bearing different aryl groups were studied, an equimolecular mixture of products was obtained (see Supplementary Fig. 30, compounds $\mathbf{6}$ and $\mathbf{6}^{\prime}$ ), indicating that the reaction takes places through a common intermediate. Then, a Friedel-Crafts reaction between the carbocation INT II and pyrrole takes place, generating the protonated intermediate III (INT III). This step is also theoretically found exergonic $(-14$ or $\left.-26.3 \mathrm{kcal} \mathrm{mol}^{-1}\right)$ and kinetically favorable $\left(E_{\mathrm{a}}=3.8 \mathrm{kcal}\right.$ $\left.\mathrm{mol}^{-1}\right)$. A final rearomatization by deprotonation of INT III gives the final product (Fig. 3d). For such deprotonation, both DIPEA and the anion acetate (formed during the reaction) would act as a base through very exergonic processes (see entries 16 and 17 from Table 1 for reactions in the presence of $\mathrm{Na}_{2} \mathrm{CO}_{3}$ and $\mathrm{LiOAc}$ ).

In order to gain a better understanding of the reaction mechanism, laser flash photolysis (LFP) measurements have been carried out. Excitation of $\mathbf{3 g}$ at $355 \mathrm{~nm}$ results in two peaks at 468 and $530 \mathrm{~nm}$ at $40 \mathrm{~ns}$ after the laser pulse, which are assigned to the $\mathbf{3} \mathbf{g}$ radical cation $\left(\mathbf{3} \mathbf{g}^{\bullet+}\right)$ and the excited triplet state of $\mathbf{3 g}\left({ }^{\mathbf{3}} \mathbf{3} \mathbf{g}^{*}\right)$, respectively (Fig. $4 \mathrm{a}$, black line, for further details, see also Supplementary Note 2). This experiment was performed in the presence of 1a to identify the possible transient reaction intermediates (Fig. $4 \mathrm{a}$, red line). Two new absorption bands at 360 and $490 \mathrm{~nm}$ are clearly observed, which correspond to intermediates I and II, respectively, based on literature data ${ }^{45}$. The lifetime of the carbocation INT II also depends on the nucleophilicity of the anionic leaving group (see Supplementary Information Fig. 4). In order to check whether formation of INT II and INT I is instantaneous with the laser pulse, additional LFP experiments of $\mathbf{3 g}$ in the presence of increasing amounts of $\mathbf{1 a}$ were performed (Fig. 4c and d). Generation of INT II is practically instantaneous even at lower concentration of 1a (Fig. 4d), whereas lifetimes of INT I are not affected by higher amounts of $\mathbf{1 a}$ (Fig. 4c). This result suggests that SET from $\mathbf{3} \mathbf{g}^{\star}$ to $\mathbf{1 a}$ at 

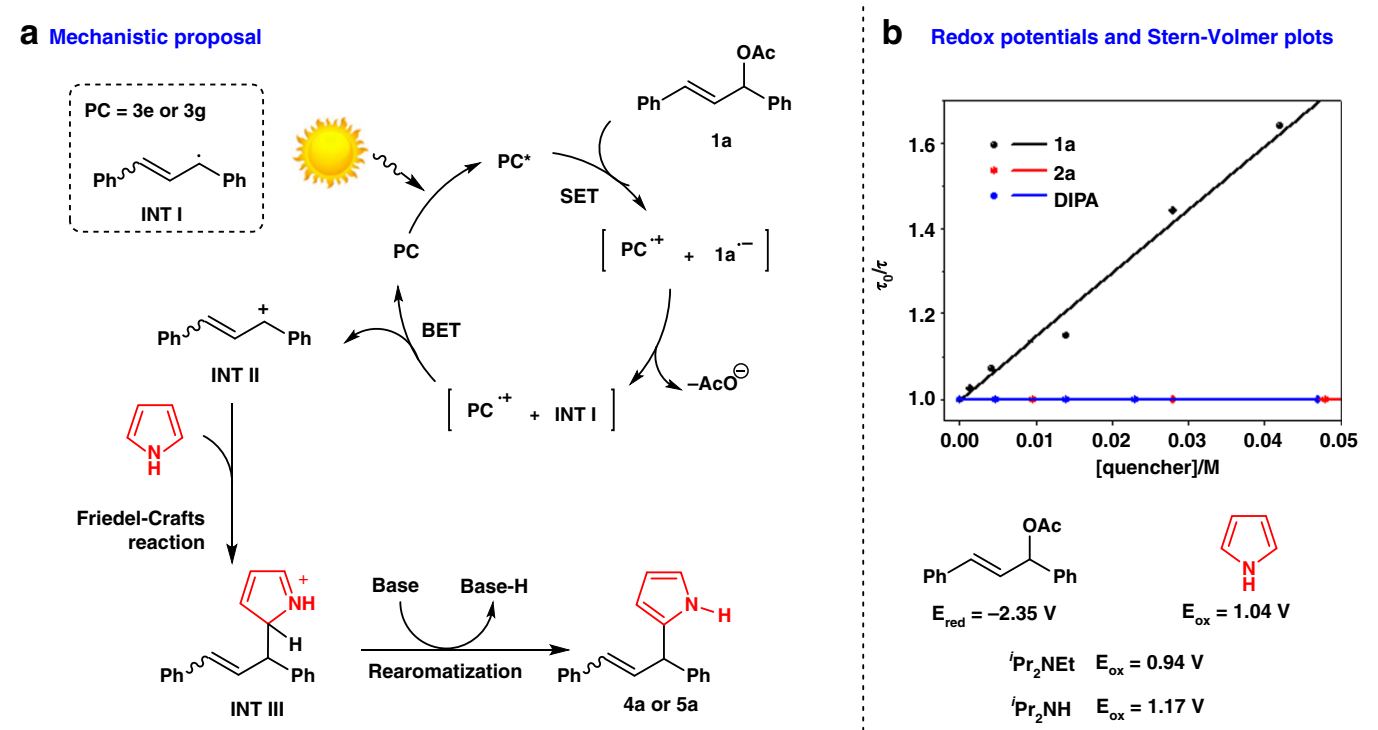

C Carbocation proof formation

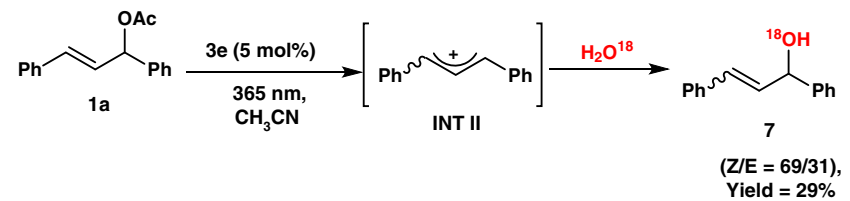

d DFT calculations

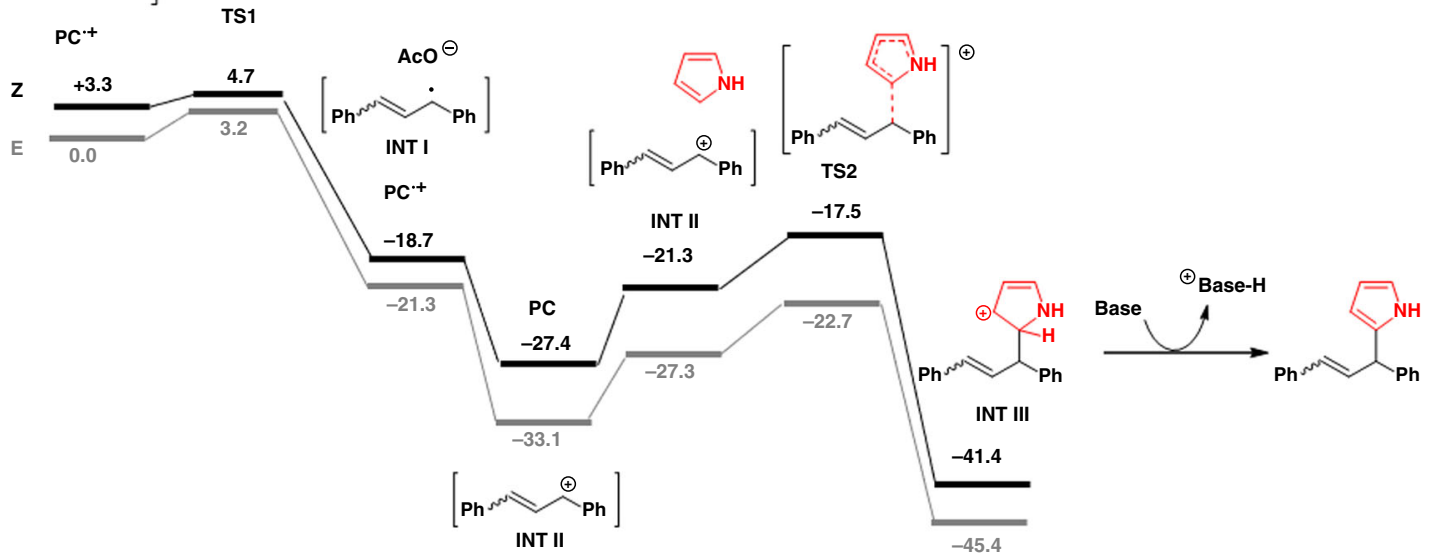

Fig. 3 Mechanistic studies. a Mechanistic proposal for the photoallylation. b Redox potentials and Stern-Volmer plots of the time-resolved fluorescence quenching of $\mathbf{3 g}$ with $\mathbf{1 a}$, pyrrole, and DIPA (DIPA = diisopropylamine). c Reaction of $\mathbf{1 a}$ with $\mathrm{H}_{2} \mathrm{O}^{18}$ under standard reaction conditions. $\mathbf{d}$ For DFT calculations, geometry optimizations were performed using the M06-2X functional in combination with the 6-311G ${ }^{\star \star}$ basis set

diffusion control rate (see Supplementary Information Fig. 9) gives rise to the contact radical ion pair at this singlet stage (Fig. 3a). All processes in the contact radical ion pairs undergo in the sub-nanosecond scale ${ }^{46}$. Fast acetate release from $\mathbf{1} \mathbf{a}^{\cdot-}$ led to INT I, which is still in close contact with $\mathbf{3 \mathbf { g } ^ { \bullet + }}$. At this point, INT I undergoes an ultrafast back electron transfer with $\mathbf{3 g}^{\cdot+}$ restoring $\mathbf{3 g}$ and generating INT II, whose amount is slightly dependent on the concentration of $1 \mathbf{a}$ in the sample (Fig. 4 d). In addition, $\mathbf{3 g}^{\bullet+}$ and INT I can split up, forming the corresponding free $\mathbf{3} \mathbf{g}^{\boldsymbol{}}+$ and free INT $\mathbf{I}$, that are detected in the LFP experiments with lifetimes in the microsecond scale
(Fig. 4a, red line). Once the detection of both intermediates I and II by LFP has been established, the question arises whether INT I or INT II (radical or carbocation) would react with a trapping agent (Fig. 4e and transient absorption spectrum in Fig. $4 \mathrm{~b}$ ). Addition of pyrrole to a $\mathbf{3 g} / \mathbf{1 a}$ mixture results only in a marked decrease of the INT II lifetime (Fig. 4g), while the band corresponding to INT I $(360 \mathrm{~nm})$ is not affected (Fig. 4f). Therefore, this experiment clearly corroborated with the previous data (Fig. 3) that the carbocation INT II is the reactive intermediate in our reaction. A quantum yield of $1.5 \%$ was found, suggesting a 
LFP: transient absorption spectra

a

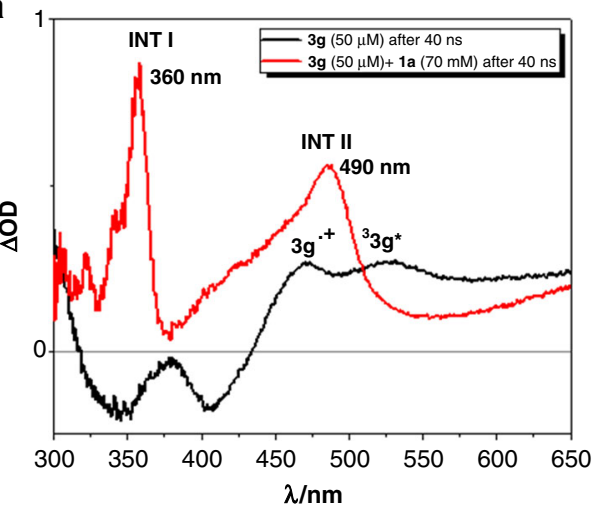

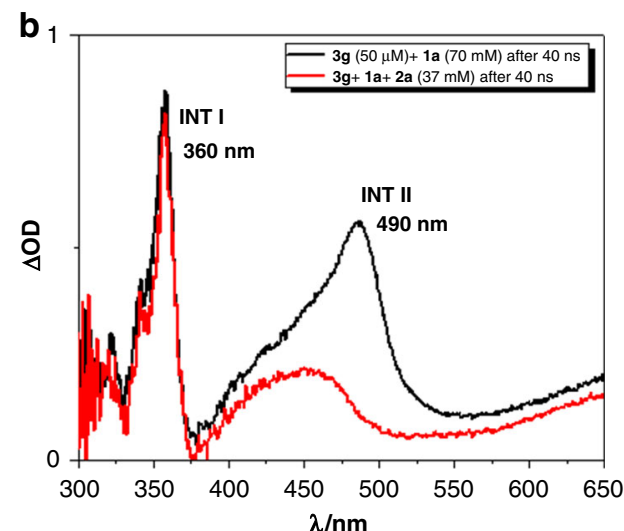

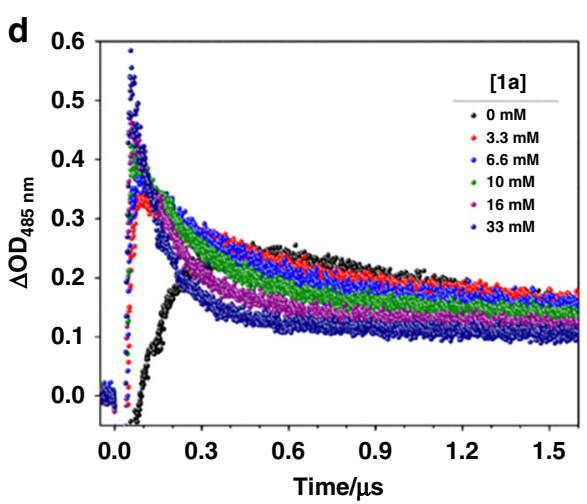

f
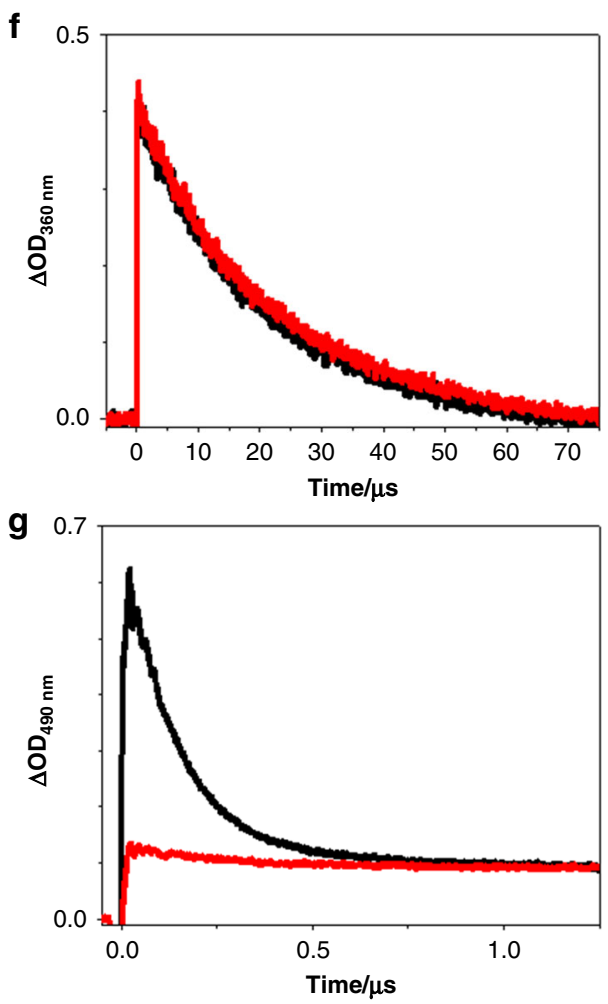

Fig. 4 Laser flash photolysis ( $\lambda_{\text {exc }}=355 \mathrm{~nm}, \mathrm{MeCN} / \mathrm{Ar}$ ) experiments. a Transient absorption spectra recorded at $40 \mathrm{~ns}$ after the laser pulse of $\mathbf{3 g}$ ( $50 \mathrm{mM}$ ) without $\mathbf{1 a}$ (black), with $70 \mathrm{mM}$ of $\mathbf{1 a}$ (red). b Transient absorption spectra recorded at $40 \mathrm{~ns}$ after the laser pulse of $\mathbf{3 g}$ ( $50 \mathrm{mM}$ ) with $70 \mathrm{mM}$ of $\mathbf{1 a}$ (black) and with $35 \mathrm{mM}$ of $\mathbf{2 a}$ (red). c Decay kinetics at $360 \mathrm{~nm}$ after 355-nm LFP of $\mathbf{3 g}$ (50 $\mu \mathrm{M}$ ) in the presence of increasing amounts of $\mathbf{1 a}$. $\mathbf{d}$ Decay kinetics at $485 \mathrm{~nm}$ after $355-\mathrm{nm}$ LFP of $\mathbf{3 g}(50 \mu \mathrm{M})$ in the presence of increasing amounts of $\mathbf{1 a}$. e Scheme of the formation of intermediate $\mathbf{I I}$ from intermediate $\mathbf{I}$ and their reaction with $\mathbf{2 a}$. $\mathbf{f}$ Lifetime of INT I: decays monitored at $360 \mathrm{~nm}$ of $\mathbf{3 g}(50 \mathrm{mM})$ and $\mathbf{1 a}(70 \mathrm{mM})$ (black line) and in the presence of $\mathbf{2 a}$ ( $37 \mathrm{mM}$ ) (red line). $\mathbf{g}$ Lifetime of INT II: decays monitored at $490 \mathrm{~nm}$ of $\mathbf{3 g}(50 \mathrm{mM})$ and $\mathbf{1 a}(70 \mathrm{mM})$ (black line) and in the presence of $\mathbf{2 a}$ (37 mM) (red line) 
Table 4 Scope of the allylation reaction with amines and alcohols for the synthesis of Z-isomers under photocatalyst $3 \mathrm{e}^{\mathrm{a}, \mathrm{b}}$
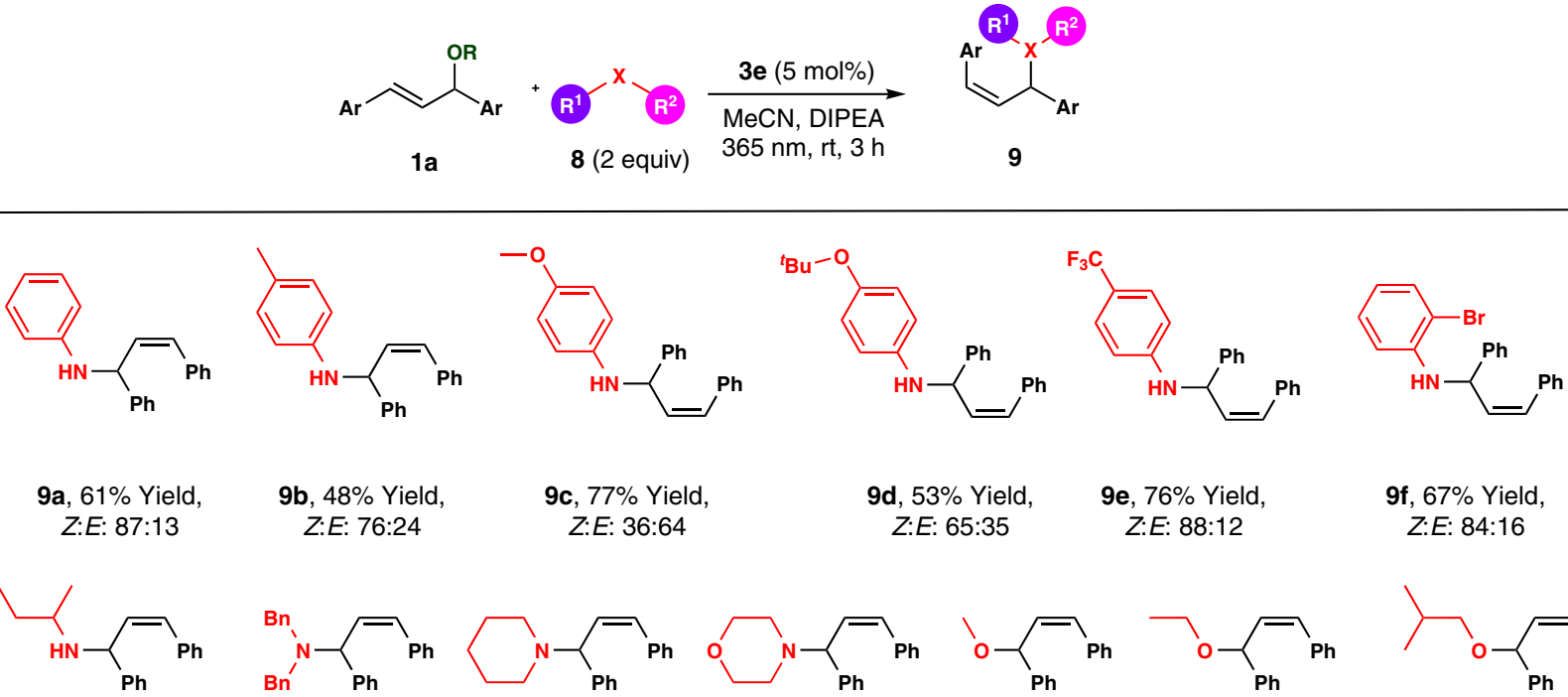

9c, $77 \%$ Yield,

9d, 53\% Yield, Z:E: $65: 35$

9e, 76\% Yield, Z:E: 88:12 Z:E: 36:64
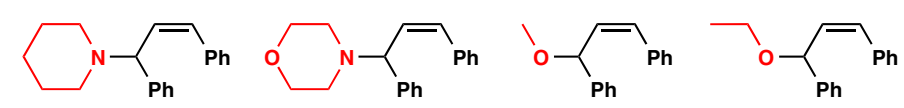

9j, 76\% Yield, Z:E: $94: 6$
9k, 76\% Yield, Z:E: 88:12

9I, 69\% Yield, Z:E: 86:14 9f, $67 \%$ Yield, Z:E: 84:16

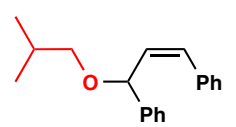

9m, 66\% Yield, Z:E: 86:14

a Conditions: 1a $(0.1 \mathrm{mmol}), \mathbf{2}(0.2 \mathrm{mmol})$, DIPEA $(0.5 \mathrm{mmol})$, and $\mathbf{3 g}(5 \mathrm{~mol} \%)$ in $\mathrm{MeCN}(1.0 \mathrm{~mL})$ blsolated yields after flash chromatography

photocatalytic process without a significant radical chain propagation $^{47}$.

Scope with alcohols and amines. Once that we proved that the reaction takes place through a carbocation intermediate formation, we decided to study other nucleophiles to prove the generality of our protocol. Allylic amines are very useful compounds that can be employed as building blocks for the synthesis of amino acids, alkaloids, and carbohydrate derivatives ${ }^{48-50}$. Moreover, this structure is present in numerous natural products and drugs with antifungal, antibacterial, and anti-inflammatory action ${ }^{51-53}$. Different amines were tried under UV irradiation in order to obtain the $Z$-allylated amines which are not accessible by other methodologies (Table 4). Aniline gave the corresponding $Z$-allylated amine 9a with high selectivity. Aromatic amines with EDGs were well tolerated (9b-d) with moderate selectivity, whereas anilines with EWGs gave significant better yield $(\mathbf{9 e})$ and $Z / E$ ratio. In addition, the presence of $\mathrm{Br}$ at the aromatic ring was tolerated without detecting the corresponding reduced product (9f). Aliphatic primary and secondary amines were also suitable for the reaction conditions $(\mathbf{9 g}-\mathbf{h})$. Cyclic allylated amine $\mathbf{9 i}$ was obtained in good selectivity $(Z: E=91: 9)$ and good yield. Moreover, the use of morpholine as a nucleophile can be employed for the synthesis of $\mathbf{9 j}$ with excellent selectivity. The preparation of allylic ethers has a great interest, as they are also present in numerous pharmaceuticals and natural products $^{54-57}$. For this reason, alcohols were employed as nucleophiles, obtaining $Z$-allylated ethers with good yields and good selectivities $(\mathbf{9 k}-\mathbf{m})^{58}$.

Using the visible-light irradiation conditions and photocatalyst $\mathbf{3 g}$ with amines and alcohols is possible to obtain the corresponding $E$-isomers (Table 5). Aromatic amines gave the corresponding allylated compounds with high selectivities and good yields with EDGs (10b-d) and EWGs (10e), or orthobromo substituents (10f). Aliphatic primary (10g) and secondary amines $(\mathbf{1 0 h}-\mathbf{j})$ were employed, keeping in all the cases high $Z / E$ selectivity. Moreover, allylated ethers can also be obtained under visible-light irradiation with excellent selectivities $(\mathbf{1 0 k}-\mathbf{m})$. A similar mechanistic scenario was found for amines and alcohols, using $p$-toluidine $\mathbf{8 b}$ as a nucleophile in the LFP and photochemical mechanistic probes (see Supplementary Fig. 9).

\section{Discussion}

In summary, a chromoselective photocatalytic approach for the allylation of indoles, pyrroles, amines, and alcohols has been developed. This approach represents a photocatalytic allylation reaction for the synthesis of demand of $Z$ - or $E$-isomers, with only two equivalents of the desired nucleophile. Therefore, under UVlight irradiation $Z$-allylated products are obtained, while the $E$ isomer is simply prepared by changing both the light source to the visible region, and the catalytic system. DFT calculations, photochemical proofs, and mechanistic experiments indicate that the most plausible mechanism involves a nucleophilic attack to an allyl-cation intermediate.

\section{Methods}

Procedure for the preparation of Z-allylic compounds. A vial equipped with a magnetic stir bar and fitted with a Teflon screw cap septum was charged with the corresponding allylic compound $1(0.1 \mathrm{mmol})$, the corresponding heterocycle, amine, or alcohol ( $0.2 \mathrm{mmol}), \mathrm{N}$-phenyl phenothiazine (1.4 mg, $5 \mathrm{~mol} \%)$, DIPEA $(86 \mu \mathrm{L}, 0.5 \mathrm{mmol})$, and acetonitrile $(1 \mathrm{~mL})$. The reaction was degassed with three freeze-pump-thaw cycles. The vial was then backfilled with $\mathrm{N}_{2}$ and stirred under $365-\mathrm{nm}$ LED irradiation $\left(8.2460 \mathrm{~W} \mathrm{~m}^{-2}\right.$ intensity; approximate distance was $2 \mathrm{~cm}$ from the vial) at $20^{\circ} \mathrm{C}$. After $3 \mathrm{~h}$, the vial was opened, the solvent evaporated, and the crude product was purified by column chromatography to give the corresponding products 4 or 9 . 
Table 5 Scope of the allylation reaction with amines and alcohols for the synthesis of E-isomers under photocatalyst $3 \mathrm{~g}^{\mathrm{a}, \mathrm{b}}$

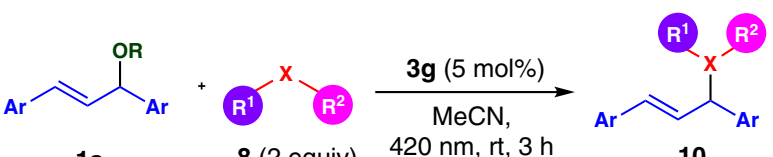

1a 8 (2 equiv) $420 \mathrm{~nm}, \mathrm{rt}, 3 \mathrm{~h} \quad 10$<smiles>C(=C/C(Nc1ccccc1)c1ccccc1)\c1ccccc1</smiles>

10a, 92\% Yield, Z:E: 9:91<smiles>CCC(C)NC(/C=C/c1ccccc1)c1ccccc1</smiles>

10g, 39\% Yield, Z:E: 7:93<smiles>Cc1ccc(NC(/C=C/c2ccccc2)c2ccccc2)cc1</smiles><smiles>COc1ccc(NC(/C=C/c2ccccc2)c2ccccc2)cc1</smiles>

10b, 84\% Yield, Z:E: 8:92<smiles>CC(C)COc1ccc(NC(/C=C/c2ccccc2)c2ccccc2)cc1</smiles>

10d, 79\% Yield, ${ }^{\mathrm{C}}$ Z:E: 3:97

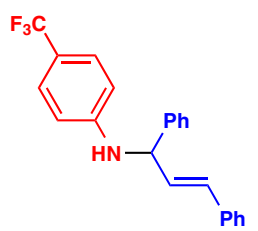

10e, $84 \%$ Yield, Z:E: 7:93

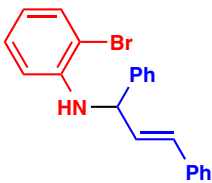

10f, $82 \%$ Yield, Z:E: $7: 93$<smiles>C(=C/C(c1ccccc1)N1CCCCC1)\c1ccccc1</smiles><smiles>C(=C/C(c1ccccc1)N1CCOCC1)\c1ccccc1</smiles><smiles>COC(/C=C/c1ccccc1)c1ccccc1</smiles><smiles>CCOC(/C=C/c1ccccc1)c1ccccc1</smiles><smiles>CC(C)COC(/C=C/c1ccccc1)c1ccccc1</smiles>

10h, 60\% Yield, 10i, 68\% Yield,

10j, 66\% Yield, Z:E: 5:95
10k, 48\% Yield, Z:E: 5:95
10I, 37\% Yield Z:E: 6:94

10m, 34\% Yield Z:E: $13: 87$

a Conditions: 1a $(0.1 \mathrm{mmol}), 8(0.2 \mathrm{mmol})$, and $\mathbf{3 g}(5 \mathrm{~mol} \%)$ in $\mathrm{MeCN}(1.0 \mathrm{~mL})$

blsolated yields after flash chromatography

'Reaction performed by adding DIPEA $(0.5 \mathrm{mmol})$

Procedure for the preparation of E-allylic compounds. A vial equipped with a magnetic stir bar and fitted with a Teflon screw cap septum was charged with the corresponding allylic compound $1(0.1 \mathrm{mmol})$, the corresponding heterocycle, amine, or alcohol (0.2 mmol), 3-(4-methoxyphenyl)-10-phenyl-10H-phenoxazine $(1.7 \mathrm{mg}, 5 \mathrm{~mol} \%)$, DIPA $(70 \mu \mathrm{L}, 0.5 \mathrm{mmol}$, only base is needed for reactions with heterocycles as nucleophile), and acetonitrile $(1 \mathrm{~mL})$. The reaction was degassed with three freeze-pump-thaw cycles. The vial was then backfilled with $\mathrm{N}_{2}$ and stirred under $420-\mathrm{nm}$ LED irradiation $\left(18.3396 \mathrm{~W} \mathrm{~m}^{-2}\right.$ intensity; approximate distance was $2 \mathrm{~cm}$ from the vial) at room temperature. After $3 \mathrm{~h}$, the vial was opened, the solvent evaporated, and the crude product was purified by column chromatography to give the corresponding products $\mathbf{5}$ or $\mathbf{1 0}$.

\section{Data availability}

The authors declare that all data supporting the findings of this study are available within the article and Supplementary Information files, and also are available from the corresponding author upon reasonable request.

Received: 30 November 2018 Accepted: 13 May 2019

Published online: 14 June 2019

\section{References}

1. Trost, B. M. \& Strege, P. E. Asymmetric induction in catalytic allylic alkylation. J. Am. Chem. Soc. 99, 1649-1651 (1977).

2. Trost, B. M. New rules of selectivity: allylic alkylations catalyzed by palladium. Acc. Chem. Res. 13, 385-393 (1980).

3. Tsuji, J., Minami, I. \& Shimizu, I. Palladium-catalyzed allylation of ketones and aldehydes with allylic carbonates via silyl enol ethers under neutral conditions. Chem. Lett. 12, 1325-1326 (1983).

4. Trost, B. M. \& Van Vranken, D. L. Asymmetric transition metal-catalyzed allylic alkylations. Chem. Rev. 96, 395-422 (1996).

5. De Meijere, A., Diederich, F. (eds) Metal-Catalyzed Cross-coupling Reactions, 2nd edn (Wiley, Weinheim, 2008).

6. D’Ischia, A., Napolitano, A. \& Pezella, A. Pyrroles and their Benzo Derivatives: Application. in Comprehensive Heterocyclic Chemistry III (eds Katritzky, A. R., Ramsden, C. A., Scriven, E. F. V. \& Taylor, R. J. K.) 353-386 (Elsevier Science, Amsterdam, 2008).
7. Malkov, A. V., Davis, S. L., Baxendale, I. R., Mitchell, W. L. \& Kočovsky, P. Molybdenum(II)-catalyzed allylation of electron-rich aromatics and heteroaromatics. J. Org. Chem. 64, 2751-2764 (1999).

8. Bandini, M., Melloni, A. \& Umani-Ronchi, A. New versatile Pd-catalyzed alkylation of indoles via nucleophilic allylic substitution: controlling the regioselectivity. Org. Lett. 6, 3199-3202 (2004).

9. Kimura, M., Futamata, M., Mukai, R. \& Tamaru, Y. Pd.catalyzed C3-selective allylation of indoles with allyl alcohols promoted by triethylborane. J. Am. Chem. Soc. 127, 4592-4593 (2005).

10. Stanley, L. M. \& Hartwig, J. F. Iridium-catalyzed regio- and enantioselective $N$-allylation of indoles. Angew. Chem. Int. Ed. 48, 7841-7844 (2009).

11. Xu, K., Gilles, T. \& Breit, B. Asymmetric synthesis of $N$-allylic indoles via regio- and enantioselective allylation of aryl hydrazines. Nat. Commun. 6, 7616 (2015)

12. Lee, J. Y., Ha, H., Bae, S., Han, I. \& Joo, J. M. Catalytic C-2 allylation of indoles by electronic modulation of the indole ring and its application to the synthesis of functionalized carbazoles. Adv. Synth. Catal. 358, 3458-3470 (2016).

13. Narayanam, J. M. R. \& Stephenson, C. R. J. Visible light photoredox catalysis: applications in organic synthesis. Chem. Soc. Rev. 40, 102-113 (2011).

14. Prier, C. K., Rankic, D. A. \& MacMillan, D. W. C. Visible light photoredox catalysis with transition metal complexes: applications in organic synthesis. Chem. Rev. 113, 5322-5363 (2013).

15. Meggers, E. Asymmetric catalysis activated by visible light. Chem. Commun. 51, 3290-3301 (2015).

16. Ravelli, D., Protti, S. \& Fagnoni, M. Carbon-carbon bond forming reactions via photogenerated intermediates. Chem. Rev. 116, 9850-9913 (2016).

17. Skubi, K. L., Blum, T. R. \& Yoon, T. P. Dual catalysis strategies in photochemical synthesis. Chem. Rev. 116, 10035-10074 (2016).

18. Pitre, S. P., McTiernan, C. D. \& Scaiano, J. C. Understanding the kinetics and spectroscopy of photoredox catalysis and transition-metal-free alternatives. Acc. Chem. Res. 49, 1320-1330 (2016).

19. Tellis, J. C. et al. Single-electron transmetalation via photoredox/nickel dual catalysis: unlocking a new paradigm for $\mathrm{sp}^{3}-\mathrm{sp}^{2}$ cross-coupling. Acc. Chem. Res. 49, 1429-1439 (2016).

20. Gentry, E. C. \& Knowles, R. R. Synthetic applications of proton-coupled electron transfer. Acc. Chem. Res. 49, 1546-1556 (2016).

21. Hernandez-Perez, A. C. \& Collins, S. Heteroleptic Cu-based sensitizers in photoredox catalysis. Acc. Chem. Res. 49, 1557-1565 (2016).

22. Goddard, J.-P., Ollivier, C. \& Fensterbank, L. Photoredox catalysis for the generation of carbon centered radicals. Acc. Chem. Res. 49, 1924-1936 (2016). 
23. Morris, S. A., Wang, J. \& Zheng, N. The prowess of photogenerated amine radical cations in cascade reactions: from carbocycles to heterocycles. Acc. Chem. Res. 49, 1957-1968 (2016).

24. Fabry, D. C. \& Rueping, M. Merging visible light photoredox catalysis with metal catalyzed C-H activations: on the role of oxygen and superoxide ions as oxidants. Acc. Chem. Res. 49, 1969-1979 (2016).

25. Majek, M. \& von Wangelin, A. J. Mechanistic perspectives on organic photoredox catalysis for aromatic substitutions. Acc. Chem. Res. 49, 2316-2327 (2016).

26. Shaw, M. H., Twilton, J. \& MacMillan, D. W. C. Photoredox catalysis in organic chemistry. J. Org. Chem. 81, 6898-6926 (2016).

27. Zhou, W.-J., Zhang, Y.-H., Gui, Y.-Y., Sun, L. \& Yu, D.-G. Merging transitionmetal catalysis with photoredox catalysis: an environmentally friendly strategy for C-H functionalization. Synthesis 50, 3359-3378 (2018).

28. Garrido-Castro, A. F., Carmen Maestro, M. \& Alemán, J. Asymmetric induction in photocatalysis-discovering a new side to light-driven chemistry. Tetrahedron Lett. 59, 1286-1294 (2018).

29. Marzo, L., Pagire, S. K., Reiser, O. \& König, B. Visible-light photocatalysis: does it make a difference in organic synthesis. Angew. Chem. Int. Ed. 57, 10034-10072 (2018).

30. Wang, C.-S., Dixneuf, P. H. \& Soule, J.-F. Photoredox catalysis for building CC bonds from $\mathrm{C}\left(\mathrm{sp}^{2}\right)-\mathrm{H}$ bonds. Chem. Rev. 118, 7532-7585 (2018).

31. Hari, D. P. \& König, B. The photocatalyzed Meerwein arylation: classic reaction of aryl diazonium salts in a new light. Angew. Chem. Int. Ed. 52, 4734-4743 (2013).

32. Ghosh, I., Marzo, L., Das, A., Shaikh, R. \& König, B. Visible-light mediated photoredox catalytic arylation reactions. Acc. Chem. Res. 49, 1566-1577 (2016).

33. Hari, D. P., Schroll, P. \& König, B. Metal-free, visible-light-mediated direct C$\mathrm{H}$ arylation of heteroarenes with aryl diazonium salts. J. Am. Chem. Soc. 134 , 2958-2961 (2012).

34. Ghosh, I., Ghosh, T., Bardagi, J. I. \& König, B. Reduction of aryl halides by consecutive visible light-induced electron transfer processes. Science 346, 725-728 (2014).

35. Meyer, A. U., Slanina, T., Yao, C.-J. \& König, B. Metal-free perfluoroarylation by visible light photoredox catalysis. ACS Catal. 6, 369-375 (2016).

36. Ghosh, I. \& König, B. Chromoselective photocatalysis: controlled bond activation through light-color regulation of redox potentials. Angew. Chem. Int. Ed. 55, 7676-7679 (2016).

37. Marzo, L., Ghosh, I., Esteban, F. \& König, B. Metal-free photocatalyzed cross coupling of bromoheteroarenes with pyrroles. ACS Catal. 6, 6780-6784 (2016).

38. Kalyani, D., McMurtrey, K. B., Neufeldt, S. R. \& Sanford, M. S. Roomtemperature $\mathrm{C}-\mathrm{H}$ arylation: merger of $\mathrm{Pd}$-catalyzed $\mathrm{C}-\mathrm{H}$ functionalization and visible-light photocatalysis. J. Am. Chem. Soc. 133, 18566-18569 (2011).

39. Zoller, J., Fabry, D. C. \& Rueping, M. Unexpected dual role of titanium dioxide in the visible light heterogeneous catalysed $\mathrm{C}-\mathrm{H}$ arylation of heteroarenes. ACS Catal. 5, 3900-3904 (2015).

40. Maity, P., Kundu, D. \& Ranu, B. C. Multigram four-step synthesis of 1,4,7triazacyclononanes with $2 \mathrm{R}_{\mathrm{a}} / \mathrm{R}_{\mathrm{b}} N$-functionalization pattern by starting from diethylenetriamine. Eur. J. Org. Chem. 2015, 1727-1734 (2015).

41. McCarthy, B. G. et al. Structure-property relationships for tailoring phenoxazines as reducing photoredox catalysts. J. Am. Chem. Soc. 140 5088-5101 (2018).

42. Roth, H. G., Romero, N. A. \& Nicewicz, D. A. Experimental and calculated electrochemical potentials of common organic molecules for applications to single-electron redox chemistry. Synlett 27, 714-723 (2016).

43. Adenier, A., Chehimi, M. M., Gallardo, I., Pinson, J. \& Vila, N. Electrochemical oxidation of aliphatic amines and their attachment to carbon and metal surfaces. Langmuir 20, 8243-8253 (2004).

44. Garrido-Castro, A. F., Choubane, H., Daaou, M., Maestro, M. C. \& Alemán, J. Asymmetric radical alkylation of $\mathrm{N}$-sulfinimines under visible light photocatalytic conditions. Chem. Commun. 53, 7764-7767 (2017).

45. Miranda, M. A., Perez-Prieto, J., Font-Sanchis, E., Kónya, K. \& Scaiano, J. C. Flash photolysis of 1,3-dichloro-1,3-diphenylpropane in polar solvents: generation of a stabilized $\gamma$-chloropropyl cation, subsequent formation of a propenyl cation, and nucleophilic trapping of both cations. J. Phys. Chem. A 102, 5724-5727 (1998).

46. Mattay, J. \& Vondenhof, M. Contact and solvent-separated radical ion pairs in organic. In Photoinduced electron transfer III. Topics in Current Chemistry (Ed. Mattay, J.) 219-255 (Springer, Heidelberg, 1991).

47. Kuhn, H. J., Braslavsky, S. E. \& Schmidt, R. Chemical actinometry (IUPAC technical report). Pure Appl. Chem. 76, 2105-2146 (2004).

48. Cheikh, R. B., Chaabouni, R., Laurent, A., Mison, P. \& Nafti, A. Synthesis of primary allylic amines. Synthesis 685-700 (1983).
49. Johannsen, M. \& Jørgensen, K. A. Allylic amination. Chem. Rev. 98, 1689-1708 (1998)

50. Trost, B. M. \& Crawley, M. L. Asymmetric transition-metal-catalyzed allylic alkylations: applications in total synthesis. Chem. Rev. 103, 2921-2943 (2003).

51. Petranyi, G., Ryder, N. S. \& Stutz, A. Allylamine derivatives: new class of synthetic antifungal agents inhibiting fungal squalene epoxidase. Science 224, 1239-1241 (1984)

52. Stutz, A. Allylamine derivatives-a new class of active substances in antifungal chemotherapy. Angew. Chem. Int. Ed. 26, 320-328 (1987).

53. Nanavati, S. M. \& Silverman, R. B. Mechanisms of inactivation of gammaaminobutyric acid aminotransferase by the antiepilepsy drug gamma-vinyl GABA (vigabatrin). J. Am. Chem. Soc. 113, 9341-9349 (1991).

54. Mizuguchi, E. \& Achiwa, K. Chiral palladium complex-catalyzed synthesis of optically active vinylchroman. Chem. Pharm. Bull. 45, 1209-1211 (1997).

55. Nicolaou, K. C. et al. Natural product-like combinatorial libraries based on privileged structures. 1. General principles and solid-phase synthesis of benzopyrans. J. Am. Chem. Soc. 122, 9939-9953 (2000).

56. Cao, B., Park, H. \& Joullié, M. M. Total synthesis of Ustiloxin D. J. Am. Chem Soc. 124, 520-521 (2002).

57. Ishibashi, H., Ishihara, K. \& Yamamoto, H. A new artificial cyclase for polyprenoids: enantioselective total synthesis of $(-)$-chromazonarol, $(+)-8$ epi-puupehedione, and (-)-11'-deoxytaondiol methyl ether. J. Am. Chem. Soc. 126, 11122-11123 (2004).

58. Pochetti, G. et al. Structural insight into peroxisome proliferator-activated receptor $\gamma$ binding of two ureidofibrate-like enantiomers by molecular dynamics, cofactor interaction analysis, and site-directed mutagenesis. J. Med. Chem. 53, 4354-4366 (2010).

\section{Acknowledgements}

Financial support from the Spanish Government (CTQ2015-64561-R), CCC-UAM (computing time), and ERC (ERC-CG, 647550, 648319) is acknowledged. L.M., R.P-R, and R.C. thank CAM for the "Atracción de Talento" fellowship. The authors thank "Comunidad de Madrid" and European Structural Funds for their financial support to FotoArt-CM project (S2018/NMT-4367). We thank Miguel Ángel Miranda for helpful discussions about photochemical mechanisms.

\section{Author contributions}

A.M.M-G and A.F. carried out the optimization and scope of the reaction. R.C. and J.L-B. carried out the scope of the reaction. L.M. performed the photocatalytic studies for mechanism elucidation. R.P-R. and V.A.P. performed the Laser Flash Photolysis studies R.M-B. carried out the DFT-computational studies. J.A. conceived the project and prepared the paper, which was edited by all other authors.

\section{Additional information}

Supplementary Information accompanies this paper at https://doi.org/10.1038/s41467 019-10441-4.

Competing interests: The authors declare no competing interests.

Reprints and permission information is available online at http://npg.nature.com/ reprintsandpermissions/

Journal peer review information: Nature Communications thanks anonymous reviewer(s) for their contribution to the peer review of this work.

Publisher's note: Springer Nature remains neutral with regard to jurisdictional claims in published maps and institutional affiliations.

Open Access This article is licensed under a Creative Commons Attribution 4.0 International License, which permits use, sharing, adaptation, distribution and reproduction in any medium or format, as long as you give appropriate credit to the original author(s) and the source, provide a link to the Creative Commons license, and indicate if changes were made. The images or other third party material in this article are included in the article's Creative Commons license, unless indicated otherwise in a credit line to the material. If material is not included in the article's Creative Commons license and your intended use is not permitted by statutory regulation or exceeds the permitted use, you will need to obtain permission directly from the copyright holder. To view a copy of this license, visit http://creativecommons.org/ licenses/by/4.0/.

(C) The Author(s) 2019 